Олейников А.И.

A.I. Oleynikov

ТЕНЗОРНО-ЛИНЕЙНЫЕ МОДЕЛИ УСТАНОВИВШЕЙСЯ ПОЛЗУЧЕСТИ

ТРАНСВЕРСАЛЬНО-ИЗОТРОПНЫХ МАТЕРИАЛОВ С РАЗНЫМИ

ХАРАКТЕРИСТИКАМИ ПРИ РАСТЯЖЕНИИ И СЖАТИИ

\title{
TENSOR LINEAR MODELS OF STATIONARY CREEP OF TRANSVERSALLY ISOTROPIC MATERIALS HAVING DIFFERENT TENSILE AND COMPRESSSION PROPERTIES
}

Олейников Александр Иванович - доктор физико-математических наук, профессор, заведующий кафедрой механики и анализа конструкций и процессов Комсомольского-на-Амуре государственного технического университета (г. Комсомольск-на-Амуре). E-mail: a.i.oleinikov@mail.ru, cvmi@knastu.ru. Mr. Alexander I. Oleynikov - Doctor of Physics and Mathematics, Head of the Department of Mechanics and Analysis of Structures and Processes, Komsomolsk-on-Amur State Technical University (Komsomolskon-Amur). E-mail: a.i.oleinikov@mail.ru, cvmi@knastu.ru.

Аннотация: Предложены простейшие определяющие соотношения для описания установившейся ползучести трансверсально-изотропных материалов с разными свойствами при растяжении и сжатии. Работа выполнена при финансовой поддержке ДВО РАН, РФФИ и Минобрнауки РФ (проекты 09-І-П11-03, 09-II-СУ-03-001, 07-01-00747, 2.1.1/ 1686).

Summary: The paper suggests the simplest defining relations for description of stationary creep of transversally isotropic materials having different tension and compression properties. The study has been done with financial support from the Far-Eastern Branch of the Russian Academy of Sciences, the Russian Fundamental Research Fund, the Ministry of Education and Science of the Russian Federation (projects 09-I-П11-03, 09-II-CУ-03-001, 07-01-00747, 2.1.1/ 1686).

Ключевые слова: ползучесть, трансверсальная изотропия, разносопротивляемость, закон течения.

Keywords: creep, transverse isotropy, heteroresistance, law of flow.

УДК 539.376

1. Пусть $x_{1}, x_{2}, x_{3}$ - прямоугольная декартова система координат. Скорость деформаций установившейся ползучести $\varepsilon=\left(\varepsilon_{i j}\right)$ зависит от текущих значений напряжений $\sigma=\left(\sigma_{i j}\right)$ и температуры. Если форма этой зависимости не меняется при повороте системы координат на произвольный угол вокруг оси $x_{3}$, то материал является трансверсально-изотропным при установившейся ползучести с плоскостью изотропии $x_{1}, x_{2}$. Трансверсальной изотропией при ползучести обладают, например, прутки и тонкие плиты из легких сплавов, используемые для шпангоутов и монолитных крыловых панелей самолетов.

Зависимость $\varepsilon \sim \sigma$ может быть потенциальной зависимостью - ассоциированным законом

$$
\varepsilon_{i j}=\frac{\partial \Phi}{\partial \sigma_{i j}}, \quad \Phi=\Phi(\sigma) .
$$

Простейшая модель ползучести для трансверсально-изотропных материалов может определяться потенциалом скоростей деформаций: 


\section{Олейников А.И.}

ТЕНЗОРНО-ЛИНЕЙНЫЕ МОДЕЛИ УСТАНОВИВШЕЙСЯ ПОЛЗУЧЕСТИ ТРАНСВЕРСАЛЬНО-ИЗОТРОПНЫХ МАТЕРИАЛОВ С РАЗНЫМИ ХАРАКТЕРИСТИКАМИ ПРИ РАСТЯЖЕНИИ И СЖАТИИ

$$
\Phi\left(\sigma_{e t}\right)=\frac{1}{n+1} \sigma_{e t}^{n+1}, \quad \sigma_{e t}=\sqrt{3 / 2 T}
$$

где $\sigma_{e t}$ - эквивалентное напряжение, обобщающее напряжение Хубера-Мизеса и не зависящее от гидростатической составляющей тензора напряжений $[1 ; 3,281]$ :

$$
\begin{gathered}
T=a J_{1}^{2}+b J_{2}+c J_{3}, \quad(a>0, b>0, c>0) \\
J_{1}=\sigma_{33}-\frac{1}{2}\left(\sigma_{11}+\sigma_{22}\right), \quad J_{2}=\left(\sigma_{11}-\sigma_{22}\right)^{2}+4{\sigma_{12}}^{2}, \quad J_{3}=\sigma_{13}{ }^{2}+\sigma_{23}{ }^{2} .
\end{gathered}
$$

Потенциальный закон течения (1.1), обобщающий теорию типа Мизеса, строится на основе потенциала (1.2) и имеет вид

$$
\begin{gathered}
\varepsilon_{11}=\frac{3}{4} \sigma_{e t}^{n-1}\left[-a\left(\sigma_{33}-\frac{1}{2}\left(\sigma_{11}+\sigma_{22}\right)+2 b\left(\sigma_{11}-\sigma_{22}\right)\right)\right], \\
\varepsilon_{22}=\frac{3}{4} \sigma_{e t}^{n-1}\left[-a\left(\sigma_{33}-\frac{1}{2}\left(\sigma_{11}+\sigma_{22}\right)-2 b\left(\sigma_{11}-\sigma_{22}\right)\right)\right], \\
\varepsilon_{33}=\frac{3}{4} \sigma_{e t}^{n-1}\left[2 a\left(\sigma_{33}-\frac{1}{2}\left(\sigma_{11}+\sigma_{22}\right)\right)\right], \\
\varepsilon_{12}=\frac{3}{4} \sigma_{e t}^{n-1} 8 b \sigma_{12}, \quad \varepsilon_{13}=\frac{3}{4} \sigma_{e t}^{n-1} 2 c \sigma_{13}, \quad \varepsilon_{23}=\frac{3}{4} \sigma_{e t}^{n-1} 2 c \sigma_{23} .
\end{gathered}
$$

Постоянные $a, b, c, n$ в (1.5) могут быть определены последовательно из опытов при чистом растяжении образцов, вырезанных вдоль осей $x_{3}, x_{1}$ и вдоль перпендикуляра к оси $x_{1}$ в плоскости $x_{2}, x_{3}$ под углом $\pi / 4$ к оси $x_{3}$ :

$$
a=\frac{2}{3} A^{\frac{2}{n+1}}, \quad b=\frac{2}{3} B^{\frac{2}{n+1}}-\frac{1}{4} a, \quad c=\frac{4}{3} C^{\frac{2}{n+1}}-\frac{1}{4} a-b,
$$

где $A, B, C, n$ - постоянные степенной аппроксимации зависимости скорости ползучести от напряжения для экспериментальных кривых ползучести, соответствующих вышеуказанным образцам.

Непотенциальный закон течения, обобщающий закон Нортона и использующий эквивалентное напряжение $\sigma_{e t}$, записывается следующим образом:

$$
\varepsilon_{i j}=\left[3\left(a J_{1}^{2}+b J_{2}+c J_{3}\right) / 2\right]^{\frac{n-1}{2}} s_{i j},
$$

где $s_{i j}=\sigma_{i j}-1 / 3 \sigma_{p q} \delta_{p q} \delta_{i j}-$ девиатор тензора напряжений и 


$$
a=\frac{2}{3}\left(\frac{3}{2} A\right)^{\frac{2}{n-1}}, \quad b=\frac{2}{3}\left(\frac{3}{2} B\right)^{\frac{2}{n-1}}-a / 4, \quad c=4\left(\frac{3}{2} C\right)^{\frac{2}{n-1}}-b-a / 4
$$

2. В достаточно широком диапазоне напряжений прутки и тонкие плиты из титановых и алюминиево-магниевых сплавов, как правило, неодинаково деформируются в процессе ползучести при растяжении и сжатии в данном одном и том же фиксированном направлении $[2,53 ; 4,181]$. У этих материалов коэффициенты ползучести $a, b, c$ и показатель ползучести $n$ в (1.5) и (1.7), определенных по растяжению и по сжатию одинаково ориентированных образцов, существенно отличаются.

Трансверсально-изотропный материал в направлении оси $x_{i}$ имеет разные характеристики ползучести при растяжении и сжатии, если в любой фиксированный момент времени значения деформаций и их скоростей в этом направлении существенно отличаются для растягивающих и сжимающих напряжений одинаковой величины $\left|\sigma_{i i}\right|$.

Предлагается следующий потенциал скоростей ползучести для трансверсальноизотропных материалов с разными характеристиками по растяжению и сжатию:

$$
\Phi=\frac{f_{1}(\xi, \eta)}{n_{1}+1} \sigma_{e t 1}^{n_{1}+1}+\frac{f_{2}(\xi, \eta)}{n_{2}+1} \sigma_{e t 2}^{n_{2}+1}
$$

где

$$
\begin{gathered}
\sigma_{e t m}=\sqrt{3 / 2 T_{m}}, \quad T_{m}=a_{m} J_{1}^{2}+b_{m} J_{2}+c_{m} J_{3},(m=1,2),\left(a_{m}>0, b_{m}>0, c_{m}>0\right), \\
\xi=\frac{J_{1}}{\sqrt{J}}, \quad \eta=\sqrt{\frac{J_{3}}{J}}, \quad\left(J=J_{1}^{2}+J_{2}+J_{3}\right), \\
f_{1}(\xi, \eta)=\sum_{k=1}^{3} \cdot \prod_{l=1}^{6} \frac{\overrightarrow{r_{l}} \cdot \overrightarrow{r_{k l}}}{\overrightarrow{r_{k l}} \cdot \overrightarrow{r_{k l}}}, \quad f_{2}(\xi, \eta)=\sum_{k=4}^{6} \cdot \prod_{l=1}^{6} \frac{\overrightarrow{r_{l}} \cdot \overrightarrow{r_{k l}}}{\overrightarrow{r_{k l}} \cdot \overrightarrow{r_{k l}}}, \\
\overrightarrow{r_{l}}=\left(\xi-\xi_{l}\right) \overrightarrow{e_{1}}+\left(\eta-\eta_{l}\right) \overrightarrow{e_{2}}, \overrightarrow{r_{k l}}=\left(\xi_{k}-\xi_{l}\right) \overrightarrow{e_{1}}+\left(\eta_{k}-\eta_{l}\right) \overrightarrow{e_{2}},\left(\overrightarrow{e_{i}} \cdot \overrightarrow{e_{j}}=\delta_{i j}\right) .
\end{gathered}
$$

Параметры $|\xi| \leq 1,0 \leq \eta \leq 1$ в соответствии с формулами (2.3) характеризуют тип напряженного состояния в данном направлении. При чистых растяжениях $\sigma_{33}>0, \sigma_{11}>0$ (или $\sigma_{22}>0$ ), а также $\sigma_{33}^{\times}>0$ соответственно вдоль осей $x_{3}, x_{1}$ (или $x_{2}$ ), а также вдоль перпендикуляра к оси $x_{1}$ в плоскости $x_{2}, x_{3}$ под углом $\pi / 4$ к оси $x_{3}$ имеем в пространстве $(\xi, \eta)$ три нижеследующие точки:

$$
\left(\xi_{1}, \eta_{1}\right)=(1,0), \quad\left(\xi_{2}, \eta_{2}\right)=(-1 / \sqrt{5}, 0), \quad\left(\xi_{3}, \eta_{3}\right)=(1 / \sqrt{13}, 2 / \sqrt{13}) .
$$

При чистых сжатиях $\sigma_{33}<0, \sigma_{11}<0$ (или $\sigma_{22}<0$ ), $\sigma_{33}^{\times}<0$ получаем следующие три точки: 


$$
\left(\xi_{4}, \eta_{4}\right)=(-1,0),\left(\xi_{5}, \eta_{5}, \zeta_{5}\right)=(1 / \sqrt{5}, 0,0),\left(\xi_{6}, \eta_{6}\right)=(-1 / \sqrt{13}, 2 / \sqrt{13})
$$

Потенциальный закон течения, обобщающий (1.5) и использующий узлы интерполяции (2.6), (2.7), строится на основе потенциала (2.1). Постоянные $a_{m}, b_{m}, c_{m}$ в (2.2) определяются по кривым ползучести, полученных при чистом растяжении $(m=1)$ и чистом сжатии $(m=2)$ вдоль указанных направлений.

Закон течения, обобщающий (1.7), имеет вид

$\varepsilon_{i j}=\left[f_{1}(\xi, \eta)\left[3\left(a_{1} J_{1}^{2}+b_{1} J_{2}+c_{1} J_{3}\right) / 2\right]^{\frac{n_{1}-1}{2}}+f_{2}(\xi, \eta)\left[3\left(a_{2} J_{1}^{2}+b_{2} J_{2}+c_{2} J_{3}\right) / 2\right]^{\frac{n_{2}-1}{2}}\right] s_{i j}$,

где

$$
\begin{gathered}
a_{1}=\frac{2}{3}\left(\frac{3}{2} B_{13}\right)^{\frac{2}{n_{1}-1}}, b_{1}=\frac{2}{3}\left(\frac{3}{2} B_{11}\right)^{\frac{2}{n_{1}-1}}-\frac{a_{1}}{4}, \quad c_{1}=4\left(\frac{3}{2} B_{1 \times}\right)^{\frac{2}{n_{1}-1}}-\frac{a_{1}}{4}-b_{1} ; \\
a_{2}=\frac{2}{3}\left(\frac{3}{2} B_{23}\right)^{\frac{2}{n_{2}-1}}, b_{2}=\frac{2}{3}\left(\frac{3}{2} B_{21}\right)^{\frac{2}{n_{2}-1}}-\frac{a_{2}}{4}, \quad c_{2}=4\left(\frac{3}{2} B_{2 \times}\right)^{\frac{2}{n_{2}-1}}-\frac{a_{2}}{4}-b_{2} .
\end{gathered}
$$

В равенствах (2.9) $B_{m 3}, B_{m 1}, B_{m \times}$ и $n_{m}$ являются коэффициентами и показателями установившейся ползучести по чистым растяжениям $(m=1)$ и сжатиям $(m=2)$ призматических образцов, вырезанных в вышеуказанных направлениях.

Если характеристики ползучести при сжатии и растяжении одинаковы, то для напряженных состояний вида (2.6) и (2.7) соответственно потенциалы (2.1) и (1.2), а также связи $(2.8)$ и (1.7) со нгадают; в других то чках $(\xi, \eta)$ их близость определяется значениями лагранжевых многочленов влияния узлов (2.6), (2.7).

При условии $a=3 \beta / 2, b=\beta / 2, c=2 \beta$, где $\beta=\frac{3}{2}\left(\frac{B_{*}}{n_{*}+1}\right)^{2 /\left(n_{*}+1\right)}$, напряжение $\sigma_{e t}$ совпадает с эквивалентным напряжением Хубера-Мизеса, а соотношения (1.5), (1.7) - с законом течения при приведенном октаэдрическом касательном напряжении и законом Нортона соответственно.

\section{ЛИТЕРАТУРА}

1. Аннин, Б.Д. Модели упругопластического деформирования трансверсально-изотропных материалов / Б.Д. Аннин // Сибирский журнал индустриальной математики. - 1999. - Т. 2. - № 2. С. $197-200$.

2. Никитенко, А.Ф. Ползучесть и длительная прочность металлических материалов / А.Ф. Никитенко. - Новосибирск: НГАСУ, 1997. - 278 с.

3. Работнов, Ю.Н. Ползучесть элементов конструкций / Ю.Н. Работнов. - М.: Наука, 1966. - 752 с.

4. Цвелодуб, И.Ю. Постулат устойчивости и его приложения в теории ползучести металлических материалов / И.Ю. Цвелодуб. - Новосибирск: ИГиЛ СО АН СССР, 1991. - 202 с. 